\title{
Vitamin E intake from natural sources and head and neck cancer risk: a pooled analysis in the International Head and Neck Cancer Epidemiology consortium
}

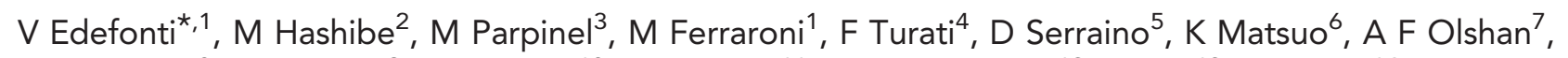
J P Zevallos ${ }^{8}$, D M Winn ${ }^{9}$, K Moysich $^{10}$, Z-F Zhang ${ }^{11}$, H Morgenstern $^{12}$, F Levi ${ }^{13}, \mathrm{~K} \mathrm{Kelsey}^{14}, \mathrm{M} \mathrm{McClean}^{15}$, C Bosetti ${ }^{16}$, S Schantz ${ }^{17}$, G-P Yu ${ }^{18}$, P Boffetta ${ }^{19}$, S-C Chuang ${ }^{20}$, Y-C A Lee ${ }^{21}$, C La Vecchia ${ }^{1}$ and A Decarli ${ }^{1,4}$ ${ }^{1}$ Laboratorio di Statistica Medica, Biometria ed Epidemiologia 'G. A. Maccacaro', Dipartimento di Scienze Cliniche e di Comunità, Università degli Studi di Milano, via A. Vanzetti, 5, 20133 Milano, Italy; ${ }^{2}$ Division of Public Health, Department of Family \& Preventive Medicine and Huntsman Cancer Institute, University of Utah School of Medicine, 375 Chipeta Way, Salt Lake City, UT 84108, USA; ${ }^{3}$ Department of Medical and Biological Sciences, University of Udine, Piazzale M. Kolbe, 4, 33100 Udine, Italy; ${ }^{4}$ S. C. Statistica Medica, Biometria e Bioinformatica, Fondazione IRCSS Istituto Nazionale Tumori di Milano, via A. Vanzetti, 5, 20133, Milano, Italy; ${ }^{5}$ Epidemiology and Biostatistics Unit, CRO Aviano National Cancer Institute, IRCCS, via F. Gallini, 2, 33081 Aviano (PN), Italy; ${ }^{6}$ Department of Preventive Medicine, Faculty of Medical Sciences, Kyushu University, 3-1-1 Maidashi, Higashi-ku, Fukuoka, 812-8582 Japan; ${ }^{7}$ University of North Carolina School of Public Health, Chapel Hill, NC, USA; ${ }^{8}$ Department of Otolaryngology/Head and Neck Surgery, University of North Carolina at Chapel Hill, 170 Manning Drive, Campus Box 7070, Chapel Hill, NC 27599-7070, USA; ${ }^{9}$ Division of Cancer Control and Population Sciences, National Cancer Institute, 9609 Medical Center Drive, Bethesda, MD 20892-9764, USA; ${ }^{10}$ Roswell Park Cancer Institute, Buffalo, NY, USA; ${ }^{11}$ Department of Epidemiology, UCLA School of Public Health, 71-225 CHS, Box 951772, Los Angeles, CA 90095-1772, USA; ${ }^{12}$ Departments of Epidemiology and Environmental Health Sciences, School of Public Health and Comprehensive Cancer Center, University of Michigan, Ann Arbor, MI, USA; ${ }^{13}$ Institute of Social and Preventive Medicine (IUMSP), Lausanne University Hospital (CHUV), Route de la Corniche 10, 1010 Lausanne, Switzerland; ${ }^{14}$ Department of Pathology and Laboratory Medicine, Brown University, 70 Ship Street, G-E5, Providence, RI 02912, USA; ${ }^{15}$ Department of Environmental Health, Boston University School of Public Health, 715 Albany Street, Talbot 4W, Boston, MA 02118, USA; ${ }^{16}$ Department of Epidemiology, IRCCS-Istituto di Ricerche Farmacologiche Mario Negri, via G. La Masa, 19, 20156 Milano, Italy; ${ }^{17}$ Department of Otolaryngology, New York Eye and Ear Infirmary, 310 E 14th Street, New York, NY 10003, USA; ${ }^{18}$ Medical Informatics Center, Peking University, Peking, China; ${ }^{19}$ The Tisch Cancer Institute and Institute of Translational Epidemiology, Icahn School of Medicine at Mount Sinai, 1425 Madison Avenue, New York, NY 10029, USA; ${ }^{20}$ Institute of Population Health Sciences, National Health Research Institutes, Zhunan, Miaoli County, Taiwan and ${ }^{21}$ Division of Public Health, Department of Family \& Preventive Medicine, University of Utah School of Medicine, 375 Chipeta Way, Salt Lake City, UT 84108, USA

Background: Evidence for the possible effect of vitamin E on head and neck cancers (HNCs) is limited.

Methods: We used individual-level pooled data from 10 case-control studies (5959 cases and 12248 controls) participating in the International Head and Neck Cancer Epidemiology (INHANCE) consortium to assess the association between vitamin E intake from natural sources and cancer of the oral cavity/pharynx and larynx. Adjusted odds ratios (ORs) and 95\% confidence

*Correspondence: Dr V Edefonti; E-mail: valeria.edefonti@unimi.it

Received 19 December 2014; revised 24 March 2015; accepted 7 April 2015; published online 19 May 2015

(c) 2015 Cancer Research UK. All rights reserved 0007-0920/15 
intervals (Cls) were estimated using unconditional logistic regression models applied to quintile categories of nonalcohol energyadjusted vitamin E intake.

Results: Intake of vitamin E was inversely related to oral/pharyngeal cancer ( $O R$ for the fifth vs the first quintile category $=0.59$, 95\% Cl: 0.49-0.71; $P$ for trend <0.001) and to laryngeal cancer (OR=0.67, 95\% Cl: 0.54-0.83, $P$ for trend <0.001). There was, however, appreciable heterogeneity of the estimated effect across studies for oral/pharyngeal cancer. Inverse associations were generally observed for the anatomical subsites of oral and pharyngeal cancer and within covariate strata for both sites.

Conclusion: Our findings suggest that greater vitamin E intake from foods may lower HNC risk, although we were not able to explain the heterogeneity observed across studies or rule out certain sources of bias.

Since the mid 1980s, evidence had been accumulating from laboratory and epidemiological studies on a putative role of antioxidant nutrients, especially $\beta$-carotene and vitamin $\mathrm{E}$, in the prevention of cancer of the oral cavity (Garewal, 1995) and cancers at other sites, in general (Chen et al, 1988; Block, 1992; Flagg et al, 1995; Schorah, 1995; Byers and Guerrero, 1995). In the absence of apparent indications of toxicity, these nutrients provided the possibility to be used widely without close medical supervision and were then the target for specific chemopreventive intervention trials, alone or in combination, to take advantage of possible synergies between them (Shklar and Schwartz, 1993). However, the results from these experiments were not in line with previous knowledge and expectations for several reasons (Bardia et al, 2008; Baumeister et al, 2009; Bjelakovic et al, 2012).

Besides these aspects, traditional single-nutrient analyses and more recent complementary ones on dietary patterns or total antioxidant capacity support the hypothesis that protection against cancer risk may derive from consumption in complex mixtures with other nutrients and bioactive compounds, as found in the matrix provided by whole foods or from combinations of foods (Edefonti et al, 2010a,b; Bravi et al, 2012; La Vecchia et al, 2013). This aspect may be particularly important for vitamin $\mathrm{E}$ intake, as this nutrient derives mainly from vegetables and oils of different origin, including olive oil.

The International Head and Neck Cancer Epidemiology (INHANCE) consortium (Hashibe et al, 2007; Conway et al, 2009; http://www.inhance.utah.edu, last accessed 15 March 2015) was established in 2004 to elucidate the aetiology of head and neck cancers (HNCs) through pooled analyses of individual-level data on HNCs on a large scale. Dietary habits have been previously investigated within the consortium. Among relevant foods and food groups, an inverse association with HNC risk was found for higher intakes of selected vegetables and seafood (Chuang et al, 2012). Another analysis focussing on dietary patterns found that a diet rich in monounsaturated, polyunsaturated and saturated fatty acids and vitamin $\mathrm{E}$ was inversely associated with oral and pharyngeal cancer and positively associated with laryngeal cancer (Edefonti et al, 2012). Results on vitamin E supplementation pointed to a weak and inconsistent inverse association of HNC risk with ever use and increased duration of use, although the oral cavity subsite showed a stronger association with supplemental vitamin $\mathrm{E}$ intake. Moreover, the highest frequency category of consumption of vitamin E supplements was associated with a small increase in risk of HNC ( $\mathrm{Li}$ et al, 2012). These inconsistencies in the results to date demonstrate the need for extra research within the consortium on the effective contribution of vitamin $\mathrm{E}$ intake from natural sources to HNC risk. Moreover, the INHANCE consortium offers the unique opportunity to clarify: (1) the independent role of vitamin $\mathrm{E}$, after the adjustment for other nutrients previously associated with $\mathrm{HNC}$ in independent singlenutrient analyses within the consortium (Edefonti et al, 2014; Leoncini et al, 2015); and (2) the combined contribution of the intakes of vitamin $\mathrm{E}$ and these nutrients, using standard approaches to model the effect of interactions between nutrients on HNC risk.

The specific goals of this analysis were: (1) to describe and account for central tendency and variation in the intakes of vitamin E from natural sources for the populations under examination; (2) to investigate the association between vitamin $\mathrm{E}$ intake and the risks of two HNC outcomes - oral and pharyngeal cancers combined and laryngeal cancer - after adjusting for several dietary and nondietary factors; (3) to explore whether effect estimates differ by cancer subsites or in subgroups of subjects, with particular attention to nonsmokers/nondrinkers of alcohol-containing beverages; (4) to explore the potential interaction effect between the intakes of vitamin E and other selected factors - putatively associated with HNC and to our main exposure (other selected nutrients, total fruit and vegetables, supplemental use of vitamin E) - on the two HNC outcomes of interest.

\section{MATERIALS AND METHODS}

Design and participants. Within the version 1.5 of the INHANCE consortium pooled data set, 10 case-control studies provided information on vitamin $\mathrm{E}$ intake derived from natural sources at the individual level (Blot et al, 1988; Schantz et al, 1997; Levi et al, 1998; Bosetti et al, 2003; Peters et al, 2005; Cui et al, 2006; Suzuki et al, 2006; Jayaprakash et al, 2006; Divaris et al, 2010; Bravi et al, 2013). Details on the individual studies, harmonisation of questionnaire data and data pooling methods for the consortium have been previously described (Galeone et al, 2014; Edefonti et al, 2014) and are reported in the Supplementary Table 1. Briefly, three of the selected studies were from Europe, six were from the United States and one from Asia. Six were hospital-based and four were population-based investigations. Study-specific questionnaires included a Food-Frequency Questionnaire (FFQ) section to assess each subject's usual diet during a reference period preceding cancer diagnosis for cases, or interview date for controls (for details on reproducibility and validity of the study-specific FFQs, see Edefonti et al, 2014). Overall, number and wording of FFQ questions were sufficiently detailed to allow for the calculation of intakes of total energy and several nutrients (U.S. Department of Health and Human Services, National Center for Health Statistics, 1982; DietSys, 1999; Applied Research Program, National Cancer Institute, 2005) through study-specific food composition databases (Dresser, 1983; Salvini et al, 1998; Resource Council, Science and Technology Agency, the Government of Japan, 2000; Gnagnarella et al, 2008; U.S. Department of Agriculture, Agricultural Research Service, 1993, 2013).

Informed consent was obtained from study subjects. The investigations were approved by the relevant institutional review boards, according to the rules adopted in each country.

Selection of subjects. Cases were included if their tumour had been classified by the original study as an invasive tumour of oral 
cavity, oropharynx, hypopharynx, oral cavity or pharynx not otherwise specified, larynx or HNC unspecified, according to the International Classification of Diseases for Oncology, 2nd edition (ICD-O-2) or the International Classification of Diseases, 9th or 10 th. Subjects with cancers of the salivary glands (ICD-O-2 codes C07-C08) or of the nasal cavity/ear/paranasal sinuses (ICD-O-2 codes C30-C31) were excluded. The ICD coding used for the classification into subsites has been specified in detail previously (Hashibe et al, 2007).

Subjects with missing information on natural vitamin $\mathrm{E}$ intake (1075 subjects from 6 studies) were removed from the original data. Subjects having an implausible ( $<500$ or $>5500 \mathrm{kcal})$ daily nonalcohol energy intake (defined as: total energy intake $(\mathrm{kcal})-100 \times$ number of drinks per day, as 1 drink per day $=$ $100 \mathrm{kcal}$ ) (343 subjects) or those having missing values (544 subjects) on nonalcohol energy intake were excluded from the analysis. Cases with missing information on the site of origin of their cancer (22 subjects, with 21 belonging to the MSKCC study) were also removed.

Thus, the present analyses included a total of 18207 subjects, with 5959 HNC cases and 12248 controls. There was a total of 1385 oral cavity cancer cases, 1653 oropharyngeal and 571 hypopharyngeal cancer cases (2224 pharyngeal cancer cases), 805 unspecified oral cavity/pharynx cases (giving a total of 4414 oral and pharyngeal cancer cases combined) and 1545 laryngeal cancer cases.

Definition of the exposure variable. We carried out preliminary checks on vitamin E definitions, reference periods of intake and measurement units across studies. In all of the studies, vitamin $\mathrm{E}$ was expressed as $\alpha$-tocopherol equivalents $=$ weighted sum of tochopherols and tochotrienols with vitamin activity. We extracted information on its intake from natural sources (i.e., no inclusion of intakes from fortified foods), and we expressed these intakes on a daily basis.

To assess the comparability of daily intakes across studies, we inspected the kernel density estimation plot (Scott, 2005) representing the study-specific empirical distributions of vitamin $\mathrm{E}$ intakes. We also compared study-specific summary statistics of vitamin $\mathrm{E}$ intakes across studies. As preliminary checks revealed differences across studies, we computed 'nonalcohol energy-adjusted' vitamin E intakes within each study, on both cases and controls, referring to the residual method (Willett and Stampfer, 1986).

Statistical analysis. Participants from all studies were grouped into five categories according to quintiles of 'nonalcohol energyadjusted' vitamin $\mathrm{E}$ intakes calculated on the overall sample. We estimated the odds ratios (ORs) and the corresponding 95\% confidence intervals (CIs) of oral and pharyngeal cancer (including oral, oropharyngeal, hypopharyngeal and unspecified oral/pharyngeal cancer), and laryngeal cancer, separately, for each quintile category (compared with the lowest reference one) using unconditional multiple logistic regression models (Hosmer and Lemeshow, 2000). Tests for linear trend were computed for all models scoring the quintiles as numbers from 1 to 5 . To adjust for potential confounders, the main analysis included the following set of variables in all the models: age, sex, education, race/ethnicity, study centre, cigarette smoking status, cigarette intensity, cigarette duration, cigar smoking status, pipe smoking status, alcohol drinking intensity and the interaction between cigarette intensity and alcohol drinking intensity (see Table 1 for categories used). For oral and pharyngeal cancer, separate analyses were conducted by anatomical subsite. For both cancers, stratified analyses were conducted by age, sex, education, geographic region, body mass index at time of interview, tobacco smoking, alcohol drinking and study (see Tables 4 and 5 for categories used, Supplementary Figure 1), and heterogeneity between strata was tested via likelihood ratio tests (Hosmer and Lemeshow, 2000).
We further investigated the potential role of other factors putatively associated with HNC and vitamin E intake on the basis of the literature, including several nutrients (saturated fats, monounsaturated fatty acids, polyunsaturated fatty acids, lutein plus xeaxanthin, total carotenoids, $\beta$-carotene equivalents, cryptoxanthin, lycopene and vitamin C) (quintile categories of adjusted nutrient intake via the residual method), total fruits and total vegetables (categories of intake based on study-specific quartiles among the controls) and supplement use of vitamin E (never/ever). For each additional covariate, we proposed a sensitivity analysis, where the aim is to explore whether the inclusion of the covariate changes the magnitude of the ORs for vitamin $\mathrm{E}$ intake, and an interaction analysis, where the aim is to assess the magnitude of a potential interaction between the additional covariate and vitamin $\mathrm{E}$ intake. We therefore fitted models with and without the extra covariate and the product (interaction) terms for that covariate and vitamin E categories; we then tested for the effects of those covariate and interaction effects using likelihood ratio tests. When the $P$-value for testing the null hypotheses of no interaction was $>0.1$, we ignored the interaction and reported results from the main-effects model. We also conducted an influence analysis in which each study was excluded one at a time to ensure that the magnitude of the overall estimates were not dependent on any specific study (Deeks et al, 2011).

In all the analyses described, when the $P$-value for heterogeneity between studies was $<0.1$, we used a mixed-effects modelling approach and replaced in the tables the fixed-effects ORs and CIs with the corresponding mixed-effects ones. We derived those estimates specifying a random intercept-random slope generalised linear mixed model (GLMM) with a logit link function and binomial family (Pinheiro and Bates, 2000).

For both fixed- and mixed-effects models, we adopted a complete-case approach to the analysis. However, as the Japan study did not provide information on education level for any participant (3495 subjects), we defined an extra category of education including all missing values to avoid the exclusion of these subjects from the analysis.

All statistical tests were two sided. Calculations were performed using the open-source statistical computing environment $\mathrm{R}$ (R Core Team, 2014), with its libraries 'Ime4' (Bates et al, 2011) and 'nnet' (Venables and Ripley, 2002), and Stata (Release 13, StataCorp LP, College Station, TX, USA).

\section{RESULTS}

Selected characteristics of cases and controls are shown in Table 1 for oral and pharyngeal cancer and for laryngeal cancer, respectively. Over $70 \%$ of cases and controls were white. The Italy Multicenter, US Multicenter and North Carolina studies contributed the largest proportion of cases of both cancer types combined. The US Multicenter provided cases of oral and pharyngeal cancer only. Cases were more likely than controls to smoke tobacco and drink alcohol and to use these products more frequently and for a greater number of years.

Table 2 gives selected descriptive statistics on raw values of vitamin $\mathrm{E}$ intake across studies and in all the studies combined. Study-specific distributions were all skewed to the right. The identified summary statistics showed very different values across studies. The Italy Multicenter, Switzerland and Milan (2006-2009) were the top three studies in terms of higher values of vitamin $\mathrm{E}$ intake, whereas the US Multicenter study provided the lowest values for all the statistics considered.

Table 3 gives separate ORs and the corresponding CIs for oral and pharyngeal combined and laryngeal cancers by quintile categories of vitamin $\mathrm{E}$ intake. For oral and pharyngeal cancer, we reported mixed-effects estimates ( $P$-value for heterogeneity 
Table 1. Distribution of cases of oral and pharyngeal cancer and laryngeal cancer and controls according to selected variables (International Head and Neck Cancer Epidemiology (INHANCE) consortium)

\begin{tabular}{|c|c|c|c|c|c|c|c|c|}
\hline & $\begin{array}{c}\text { Oral and } \\
\text { pharyngeal } \\
\text { cases }\end{array}$ & (\%) & Controls & $(\%)$ & $\begin{array}{l}\text { Laryngeal } \\
\text { cases }\end{array}$ & (\%) & Controls & (\%) \\
\hline \multicolumn{9}{|l|}{ Age (years) } \\
\hline$<40$ & 208 & 4.7 & 681 & 5.6 & 26 & 1.7 & 681 & 5.6 \\
\hline$\geqslant 40$ to $\leqslant 44$ & 194 & 4.4 & 563 & 4.6 & 45 & 2.9 & 563 & 4.6 \\
\hline$\geqslant 45$ to $\leqslant 49$ & 446 & 10.1 & 949 & 7.7 & 123 & 8.0 & 949 & 7.7 \\
\hline$\geqslant 50$ to $\leqslant 54$ & 645 & 14.6 & 1731 & 14.1 & 188 & 12.2 & 1731 & 14.1 \\
\hline$\geqslant 55$ to $\leqslant 59$ & 816 & 18.5 & 2079 & 17.0 & 271 & 17.5 & 2079 & 17.0 \\
\hline$\geqslant 60$ to $\leqslant 64$ & 713 & 16.2 & 2029 & 16.6 & 290 & 18.8 & 2029 & 16.6 \\
\hline$\geqslant 65$ to $\leqslant 69$ & 658 & 14.9 & 1931 & 15.8 & 279 & 18.1 & 1931 & 15.8 \\
\hline$\geqslant 70$ to $\leqslant 74$ & 474 & 10.7 & 1540 & 12.6 & 227 & 14.7 & 1540 & 12.6 \\
\hline$\geqslant 75$ & 260 & 5.9 & 743 & 6.1 & 96 & 6.2 & 743 & 6.1 \\
\hline Missing & 0 & 0.0 & 2 & 0.0 & 0 & 0.0 & 2 & 0.0 \\
\hline$\chi^{2}(P \text {-value })^{a}$ & \multicolumn{4}{|c|}{$42.0(<0.001)$} & \multicolumn{4}{|c|}{$66.5(<0.001)$} \\
\hline \multicolumn{9}{|l|}{ Sex } \\
\hline Female & 1187 & 26.9 & 3541 & 28.9 & 244 & 15.8 & 3541 & 28.9 \\
\hline Male & 3223 & 73.0 & 8702 & 71.0 & 1300 & 84.1 & 8702 & 71.0 \\
\hline Missing & 4 & 0.1 & 5 & 0.0 & 1 & 0.1 & 5 & 0.0 \\
\hline$\chi^{2}(P \text {-value })^{a}$ & \multicolumn{4}{|c|}{$6.3(0.012)$} & \multicolumn{4}{|c|}{$117.8(<0.001)$} \\
\hline \multicolumn{9}{|l|}{ Race } \\
\hline Black & 387 & 8.8 & 535 & 4.4 & 116 & 7.5 & 535 & 4.4 \\
\hline Others (with Asians) & 463 & 10.5 & 3089 & 25.2 & 101 & 6.5 & 3089 & 25.2 \\
\hline White (with Hispanics) & 3555 & 80.5 & 8596 & 70.2 & 1324 & 85.7 & 8596 & 70.2 \\
\hline Missing & 9 & 0.2 & 28 & 0.2 & 4 & 0.3 & 28 & 0.2 \\
\hline$\chi^{2}(P \text {-value })^{a}$ & \multicolumn{4}{|c|}{$491.5(<0.001)$} & \multicolumn{4}{|c|}{$281.7(<0.001)$} \\
\hline \multicolumn{9}{|l|}{ Study name } \\
\hline Boston & 313 & 7.1 & 611 & 5.0 & 71 & 4.6 & 611 & 5.0 \\
\hline Buffalo & 396 & 9.0 & 1190 & 9.7 & 168 & 10.9 & 1190 & 9.7 \\
\hline \multicolumn{9}{|l|}{ Italy Multicenter } \\
\hline Milan & 169 & 3.8 & 621 & 5.1 & 24 & 1.6 & 621 & 5.1 \\
\hline Pordenone & 471 & 10.7 & 1528 & 12.5 & 409 & 26.5 & 1528 & 12.5 \\
\hline Latina & 95 & 2.2 & 425 & 3.5 & 0 & 0.0 & 425 & 3.5 \\
\hline Japan (2001-2005) & 407 & 9.2 & 3002 & 24.5 & 86 & 5.6 & 3002 & 24.5 \\
\hline Los Angeles & 246 & 5.6 & 828 & 6.8 & 60 & 3.9 & 828 & 6.8 \\
\hline Milan (2006-2009) & 131 & 3.0 & 691 & 5.6 & 200 & 12.9 & 691 & 5.6 \\
\hline MSKCC & 74 & 1.7 & 123 & 1.0 & 32 & 2.1 & 123 & 1.0 \\
\hline North Carolina (2002-2006) & 687 & 15.6 & 1120 & 9.1 & 374 & 24.2 & 1120 & 9.1 \\
\hline Switzerland & 367 & 8.3 & 877 & 7.2 & 121 & 7.8 & 877 & 7.2 \\
\hline \multicolumn{9}{|l|}{ US Multicenter } \\
\hline Atlanta & 129 & 2.9 & 134 & 1.1 & 0 & 0.0 & 134 & 1.1 \\
\hline New Jersey & 467 & 10.6 & 459 & 3.7 & 0 & 0.0 & 459 & 3.7 \\
\hline Los Angeles & 398 & 9.0 & 501 & 4.1 & 0 & 0.0 & 501 & 4.1 \\
\hline San Francisco & 64 & 1.4 & 138 & 1.1 & 0 & 0.0 & 138 & 1.1 \\
\hline$\chi^{2}(P \text {-value })^{a}$ & \multicolumn{4}{|c|}{$1121.5(<0.001)$} & & 1092. & $0.001)$ & \\
\hline Education & & & & & & & & \\
\hline$\leqslant$ Junior high school & 863 & 19.6 & 2723 & 22.2 & 603 & 39.0 & 2723 & 22.2 \\
\hline Some high school & 885 & 20.0 & 1240 & 10.1 & 258 & 16.7 & 1240 & 10.1 \\
\hline High school graduate & 588 & 13.3 & 1267 & 10.3 & 237 & 15.3 & 1267 & 10.3 \\
\hline Technical school, some college & 1174 & 26.6 & 2305 & 18.8 & 214 & 13.9 & 2305 & 18.8 \\
\hline$\geqslant$ College graduate & 491 & 11.1 & 1703 & 13.9 & 145 & 9.4 & 1703 & 13.9 \\
\hline Missing & 413 & 9.4 & 3010 & 24.6 & 88 & 5.7 & 3010 & 24.6 \\
\hline$\chi^{2}(P \text {-value })^{a}$ & & & $0.001)$ & & & 503.7 & $.001)$ & \\
\hline Cigarette smoking status & & & & & & & & \\
\hline Never & 806 & 18.3 & 4868 & 39.7 & 90 & 5.8 & 4868 & 39.7 \\
\hline Former & 1387 & 31.4 & 4330 & 35.4 & 707 & 45.8 & 4330 & 35.4 \\
\hline Current & 2210 & 50.1 & 2986 & 24.4 & 735 & 47.6 & 2986 & 24.4 \\
\hline Missing & 11 & 0.2 & 64 & 0.5 & 13 & 0.8 & 64 & 0.5 \\
\hline$\chi^{2}(P \text {-value })^{a}$ & & 113 & $0.001)$ & & & 755.3 & $.001)$ & \\
\hline Cigarette intensity (cigaret & per day) & & & & & & & \\
\hline Never smoker & 806 & 18.3 & 4868 & 39.7 & 91 & 5.9 & 4868 & 39.7 \\
\hline$>0$ to $\leqslant 10$ & 471 & 10.7 & 1949 & 15.9 & 149 & 9.6 & 1949 & 15.9 \\
\hline$>10$ to $\leqslant 20$ & 1466 & 33.2 & 3169 & 25.9 & 628 & 40.6 & 3169 & 25.9 \\
\hline$>20$ & 1633 & 37.0 & 2137 & 17.4 & 661 & 42.8 & 2137 & 17.4 \\
\hline Missing & 38 & 0.9 & 125 & 1.0 & 16 & 1.0 & 125 & 1.0 \\
\hline$\chi^{2}(P \text {-value })^{a}$ & & 111 & $0.001)$ & & & 1015. & $0.001)$ & \\
\hline
\end{tabular}




\begin{tabular}{|c|c|c|c|c|c|c|c|c|}
\hline & $\begin{array}{c}\text { Oral and } \\
\text { pharyngeal } \\
\text { cases }\end{array}$ & $(\%)$ & Controls & $(\%)$ & $\begin{array}{l}\text { Laryngeal } \\
\text { cases }\end{array}$ & $(\%)$ & Controls & (\%) \\
\hline \multicolumn{9}{|c|}{ Duration of cigarette smoking (years) } \\
\hline Never smoker & 806 & 18.3 & 4868 & 39.7 & 91 & 5.9 & 4868 & 39.7 \\
\hline$>0$ to $\leqslant 20$ & 443 & 10.0 & 2166 & 17.7 & 102 & 6.6 & 2166 & 17.7 \\
\hline$>20$ & 3132 & 71.0 & 5123 & 41.8 & 1343 & 86.9 & 5123 & 41.8 \\
\hline Missing & 33 & 0.7 & 91 & 0.7 & 9 & 0.6 & 91 & 0.7 \\
\hline$\chi^{2}(P \text {-value })^{a}$ & \multicolumn{4}{|c|}{$1116.8(<0.001)$} & \multicolumn{4}{|c|}{$1133.7(<0.001)$} \\
\hline \multicolumn{9}{|l|}{ Cigar smoking } \\
\hline Never cigar user & 3583 & 81.2 & 8545 & 69.8 & 1323 & 85.6 & 8545 & 69.8 \\
\hline $\begin{array}{l}\text { Ever smoked } \geqslant 100 \text { cigars in a } \\
\text { lifetime }\end{array}$ & 394 & 8.9 & 636 & 5.2 & 118 & 7.6 & 636 & 5.2 \\
\hline Missing & 437 & 9.9 & 3067 & 25.0 & 104 & 6.7 & 3067 & 25.0 \\
\hline$\chi^{2}(P \text {-value })^{a}$ & \multicolumn{4}{|c|}{$33.7(0.008)$} & \multicolumn{4}{|c|}{$2.8(0.093)$} \\
\hline \multicolumn{9}{|l|}{ Pipe smoking } \\
\hline Never pipe user & 3579 & 81.1 & 8327 & 68.0 & 1325 & 85.8 & 8327 & 68.0 \\
\hline $\begin{array}{l}\text { Ever smoked } \geqslant 100 \text { pipes in a } \\
\text { lifetime }\end{array}$ & 399 & 9.0 & 864 & 7.1 & 115 & 7.4 & 864 & 7.1 \\
\hline Missing & 436 & 9.9 & 3057 & 25.0 & 105 & 6.8 & 3057 & 25.0 \\
\hline$\chi^{2}(P \text {-value })^{a}$ & \multicolumn{4}{|c|}{$1.2(0.027)$} & \multicolumn{4}{|c|}{$2.8(0.094)$} \\
\hline \multicolumn{9}{|c|}{ Alcohol consumption (drinks per day) } \\
\hline Never drinker & 548 & 12.4 & 3156 & 25.8 & 187 & 12.1 & 3156 & 25.8 \\
\hline$<1$ & 1030 & 23.3 & 4022 & 32.8 & 250 & 16.2 & 4022 & 32.8 \\
\hline$\geqslant 1$ to 3 & 973 & 22.0 & 2934 & 24.0 & 344 & 22.3 & 2934 & 24.0 \\
\hline$\geqslant 3$ to 5 & 647 & 14.7 & 1215 & 9.9 & 250 & 16.2 & 1215 & 9.9 \\
\hline$\geqslant 5$ & 1216 & 27.5 & 921 & 7.5 & 514 & 33.3 & 921 & 7.5 \\
\hline$\chi^{2}(P \text {-value })^{a}$ & \multicolumn{4}{|c|}{$1442.0(<0.001)$} & \multicolumn{4}{|c|}{$1155.2(<0.001)$} \\
\hline
\end{tabular}

Table 2. Descriptive statistics on raw values of vitamin $E$ intake ( $\mathrm{mg}$ per day) across studies and in all the studies combined (International Head and Neck Cancer Epidemiology (INHANCE) consortium)

\begin{tabular}{|l|r|r|r|r|}
\hline Study name & $\mathbf{2 0 \%}$ & Median & Mean & $\mathbf{8 0 \%}$ \\
\hline Boston & 5.37 & 7.91 & 9.00 & 11.58 \\
\hline Buffalo & 4.47 & 6.90 & 7.78 & 10.45 \\
\hline Italy Multicenter & 10.16 & 14.08 & 15.17 & 19.31 \\
\hline Japan (2001-2005) & 6.08 & 7.42 & 7.77 & 9.26 \\
\hline Los Angeles & 4.46 & 6.50 & 7.51 & 9.42 \\
\hline Milan (2006-2009) & 8.85 & 11.98 & 12.76 & 16.41 \\
\hline MSKCC & 5.05 & 7.22 & 8.84 & 11.34 \\
\hline North Carolina (2002-2006) & 4.95 & 7.29 & 8.04 & 10.64 \\
\hline Switzerland & 9.73 & 12.90 & 13.49 & 16.84 \\
\hline US Multicenter & 3.43 & 4.60 & 4.88 & 6.21 \\
\hline All studies combined & 5.37 & 8.30 & 9.73 & 13.48 \\
\hline Abbreviation: MSKCC=Memorial Sloan Kettering Cancer Center. \\
\hline
\end{tabular}

between studies $=0.011$, whereas for laryngeal cancer we reported the fixed-effects ones ( $P$-value for heterogeneity between studies $=0.464)$. Vitamin $\mathrm{E}$ intake was inversely related to oral and pharyngeal cancer, with an OR of 0.59 (95\% CI: 0.49-0.71) for the fifth quintile compared with the first one $(P$-value for trend $<0.001)$. Similarly, the OR for laryngeal cancer was 0.67 (95\% CI: 0.54-0.83) for the highest quintile category, with a $P$-value for trend $<0.001$.

Decreasing ORs with higher intakes of vitamin E were observed across oral and pharyngeal cancer subsites: $\mathrm{OR}=0.48$ (95\% CI: 0.34-0.66) for oral cavity, $\mathrm{OR}=0.63$ (95\% CI: $0.53-0.75)$ for oropharynx and hypopharynx combined and OR $=0.57$ (95\% CI: 0.41-0.78) for oral cavity or pharynx not otherwise specified
(Supplementary Table 2). The ORs for the oropharynx were similar to those of the hypopharynx site (for instance, for the fifth $v s$ the first quintile category, $\mathrm{OR}=0.66,95 \% \mathrm{CI}: \quad 0.60-0.80$ and $\mathrm{OR}=0.62$, 95\% CI: $0.47-0.83$, respectively) (data not shown) but, given the limited number of hypopharyngeal cancer cases, we decided to combine the results of these subsites.

Table 4 shows the ORs of oral and pharyngeal cancer in strata of selected variables. No appreciable heterogeneity was detected for vitamin $\mathrm{E}$ intake across strata, with consistent inverse associations for the third quintile category onwards for all the examined strata. However, in strata of tobacco consumption, a more marked protective association was evident for current smokers in the second and third quintile categories, as compared with non/exsmokers $(P$-value for heterogeneity across strata $<0.001)$. An appreciable heterogeneity between studies was found for several strata.

Table 5 shows the ORs of laryngeal cancer in strata of selected variables. No appreciable heterogeneity was found for vitamin $\mathrm{E}$ intake across strata. An indication of a stronger protective association was evident in the highest quintile category for subjects living in Europe, as compared with those living in the United States or Asia. No appreciable heterogeneity was found between studies in most of the strata.

In the interaction analyses including one extra nutrient among the selected ones (saturated fats, monounsaturated fatty acids, polyunsaturated fatty acids, lutein plus xeaxanthin, total carotenoids, betacarotene equivalents, cryptoxanthin, lycopene and vitamin C) or total fruits/total vegetables or supplement use of vitamin $\mathrm{E}$, no appreciable interaction effect was found between the additional covariate under examination and natural vitamin $\mathrm{E}$ for either cancer site.

In the sensitivity analyses including one extra nutrient at a time, likelihood ratio tests pointed to introduce the extra adjustment in the model for eight out of the nine selected nutrients for oral and 
Table 3. Odds ratios $(\mathrm{ORs})^{\mathrm{a}}$ of oral and pharyngeal combined and laryngeal cancers and corresponding confidence intervals $(95 \%$ $\mathrm{Cls}$ ) on vitamin E intake quintile categories (International Head and Neck Cancer Epidemiology (INHANCE) consortium)

\begin{tabular}{|c|c|c|c|c|c|c|c|c|}
\hline & $\begin{array}{c}\text { Oral and } \\
\text { pharyngeal cases }\end{array}$ & Controls & OR $(95 \% \mathrm{Cl})^{\mathrm{b}}$ & $P_{\text {studies }}{ }^{\mathrm{c}}$ & $\begin{array}{l}\text { Laryngeal } \\
\text { cases }\end{array}$ & Controls & OR $(95 \% \mathrm{Cl})^{\mathrm{b}}$ & $P_{\text {studies }}{ }^{\mathrm{c}}$ \\
\hline I Quintile & 976 & 1479 & 1 (Reference) & 0.011 & 315 & 1479 & 1 (Reference) & 0.464 \\
\hline II Quintile & 788 & 1832 & $0.79(0.69-0.90)$ & & 280 & 1832 & $0.94(0.76-1.16)$ & \\
\hline III Quintile & 704 & 1944 & $0.65(0.56-0.74)$ & & 248 & 1944 & $0.75(0.60-0.93)$ & \\
\hline IV Quintile & 707 & 1922 & $0.64(0.55-0.74)$ & & 298 & 1922 & $0.93(0.75-1.14)$ & \\
\hline V Quintile & 719 & 1819 & $0.59(0.49-0.71)$ & & 261 & 1819 & $0.67(0.54-0.83)$ & \\
\hline$P_{\text {for linear trend }}$ & & & $<0.001$ & & & & $<0.001$ & \\
\hline \multicolumn{9}{|c|}{ 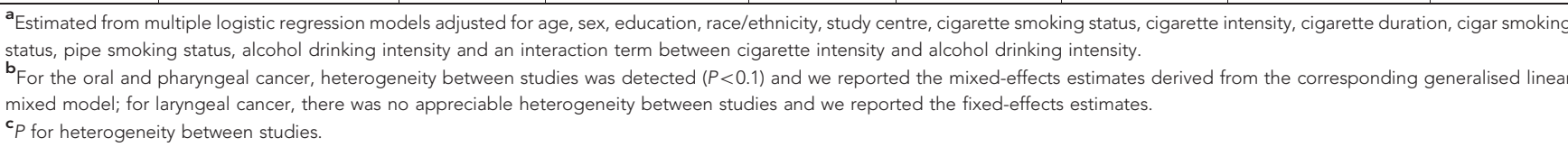 } \\
\hline
\end{tabular}

Table 4. Odds ratios (ORs) ${ }^{a, b}$ of oral and pharyngeal cancers combined and corresponding confidence intervals (95\% Cls) on vitamin $\mathrm{E}$ intake quintile categories in strata of selected covariates (International Head and Neck Cancer Epidemiology (INHANCE) consortium)

\begin{tabular}{|c|c|c|c|c|c|}
\hline & $\begin{array}{l}\text { II Quintile } \\
\text { OR (95\% Cl) }\end{array}$ & $\begin{array}{l}\text { III Quintile } \\
\text { OR (95\% Cl) }\end{array}$ & $\begin{array}{l}\text { IV Quintile } \\
\text { OR (95\% Cl) }\end{array}$ & $\begin{array}{l}\text { V Quintile } \\
\text { OR }(95 \% \mathrm{Cl})\end{array}$ & $P_{\text {studies }}{ }^{\mathrm{c}}$ \\
\hline \multicolumn{6}{|l|}{ Age (years) } \\
\hline$<55$ & $0.74(0.59-0.93)$ & $0.61(0.47-0.78)$ & $0.67(0.53-0.84)$ & $0.67(0.50-0.90)$ & 0.006 \\
\hline$\geqslant 55$ & $0.80(0.68-0.95)$ & $0.66(0.56-0.78)$ & $0.61(0.51-0.75)$ & $0.56(0.43-0.72)$ & 0.004 \\
\hline$P_{\text {strata }}{ }^{\mathrm{d}}$ & \multicolumn{5}{|c|}{0.739} \\
\hline \multicolumn{6}{|l|}{ Sex } \\
\hline Female & $0.87(0.66-1.16)$ & $0.61(0.46-0.81)$ & $0.61(0.46-0.81)$ & $0.72(0.53-0.99)$ & 0.007 \\
\hline Male & $0.77(0.66-0.90)$ & $0.67(0.57-0.80)$ & $0.67(0.57-0.79)$ & $0.54(0.43-0.68)$ & 0.109 \\
\hline$P_{\text {strata }}{ }^{\mathbf{d}}$ & \multicolumn{5}{|c|}{0.114} \\
\hline \multicolumn{6}{|l|}{ Education } \\
\hline$\leqslant$ High school graduate & $0.80(0.67-0.95)$ & $0.65(0.52-0.80)$ & $0.66(0.55-0.79)$ & $0.54(0.41-0.71)$ & 0.010 \\
\hline$\geqslant$ Some college & $0.74(0.60-0.91)$ & $0.65(0.53-0.80)$ & $0.64(0.52-0.79)$ & $0.68(0.55-0.85)$ & 0.170 \\
\hline$P_{\text {strata }}{ }^{\mathbf{d}}$ & \multicolumn{5}{|c|}{0.587} \\
\hline \multicolumn{6}{|l|}{ Geographic region ${ }^{e}$} \\
\hline Europe & $0.78(0.62-0.99)$ & $0.57(0.44-0.74)$ & $0.68(0.46-1.01)$ & $0.51(0.31-0.83)$ & $<0.001$ \\
\hline America & $0.77(0.66-0.91)$ & $0.69(0.58-0.81)$ & $0.63(0.53-0.74)$ & $0.63(0.52-0.76)$ & 0.393 \\
\hline Asia & $0.66(0.48-0.92)$ & $0.62(0.45-0.86)$ & $0.60(0.43-0.84)$ & $0.47(0.33-0.65)$ & NE \\
\hline$P_{\text {strata }}{ }^{\mathrm{d}}$ & \multicolumn{5}{|c|}{0.322} \\
\hline \multicolumn{6}{|l|}{ Body mass index } \\
\hline$<25 \mathrm{~kg} \mathrm{~m}^{-2}$ & $0.76(0.63-0.93)$ & $0.59(0.48-0.73)$ & $0.64(0.53-0.79)$ & $0.55(0.44-0.69)$ & 0.321 \\
\hline$\geqslant 25 \mathrm{~kg} \mathrm{~m}^{-2}$ & $0.79(0.65-0.95)$ & $0.72(0.57-0.89)$ & $0.68(0.56-0.83)$ & $0.67(0.53-0.84)$ & 0.030 \\
\hline$P_{\text {strata }}{ }^{\mathrm{d}}$ & \multicolumn{5}{|c|}{0.434} \\
\hline \multicolumn{6}{|l|}{ Tobacco consumption } \\
\hline Never user & $1.00(0.75-1.32)$ & $0.77(0.57-1.04)$ & $0.65(0.47-0.88)$ & $0.58(0.42-0.80)$ & 0.697 \\
\hline Former user & $0.96(0.74-1.23)$ & $0.76(0.59-0.98)$ & $0.72(0.55-0.94)$ & $0.68(0.52-0.89)$ & 0.295 \\
\hline Current user & $0.62(0.50-0.76)$ & $0.52(0.42-0.64)$ & $0.61(0.50-0.76)$ & $0.58(0.47-0.72)$ & 0.115 \\
\hline$P_{\text {strata }}{ }^{\mathbf{d}}$ & \multicolumn{5}{|c|}{$<0.001$} \\
\hline \multicolumn{6}{|l|}{ Alcohol consumption ${ }^{f}$} \\
\hline Never/light drinker & $0.88(0.72-1.07)$ & $0.71(0.58-0.88)$ & $0.79(0.64-0.96)$ & $0.72(0.57-0.90)$ & 0.225 \\
\hline Moderate drinker & $0.70(0.54-0.90)$ & $0.63(0.50-0.79)$ & $0.59(0.47-0.75)$ & $0.58(0.42-0.78)$ & 0.008 \\
\hline Heavy drinker & $0.71(0.50-1.01)$ & $0.55(0.38-0.79)$ & $0.47(0.32-0.69)$ & $0.43(0.31-0.59)$ & 0.104 \\
\hline$P_{\text {strata }}{ }^{\mathbf{d}}$ & \multicolumn{5}{|c|}{0.414} \\
\hline \multicolumn{6}{|c|}{ 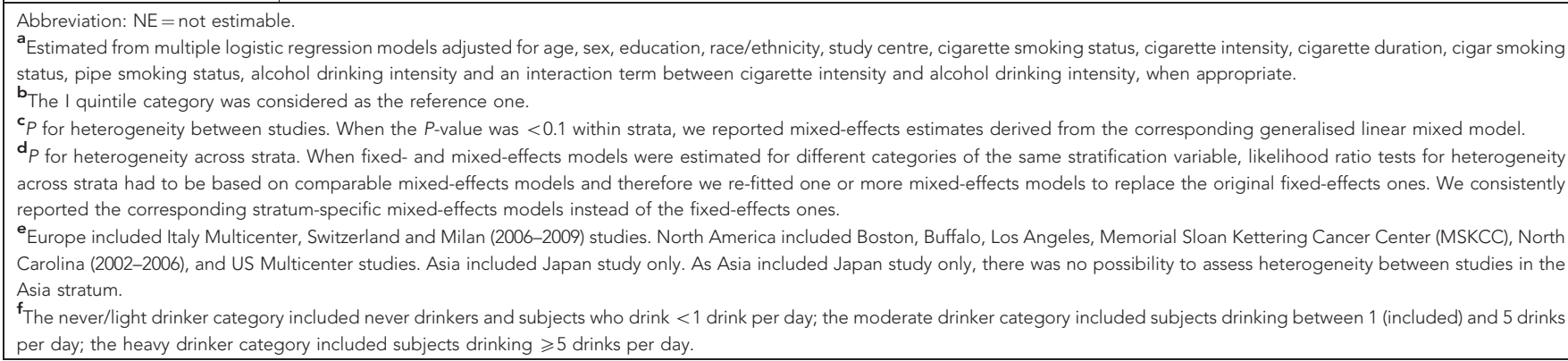 } \\
\hline
\end{tabular}


Table 5. Odds ratios (ORs) ${ }^{a, b}$ of laryngeal cancer and corresponding confidence intervals ( $95 \%$ Cls) on vitamin $\mathrm{E}$ intake quintile categories in strata of selected covariates (International Head and Neck Cancer Epidemiology (INHANCE) consortium)

\begin{tabular}{|c|c|c|c|c|c|}
\hline & $\begin{array}{c}\text { II Quintile } \\
\text { OR (95\% Cl) }\end{array}$ & $\begin{array}{l}\text { III Quintile } \\
\text { OR }(95 \% \mathrm{Cl})\end{array}$ & $\begin{array}{l}\text { IV Quintile } \\
\text { OR }(95 \% \mathrm{Cl})\end{array}$ & $\begin{array}{c}\text { V Quintile } \\
\text { OR }(95 \% \mathrm{Cl})\end{array}$ & $P_{\text {studies }}{ }^{\mathrm{c}}$ \\
\hline \multicolumn{6}{|l|}{ Age (years) } \\
\hline$<55$ & $0.98(0.66-1.47)$ & $0.66(0.43-1.01)$ & $0.67(0.44-1.02)$ & $0.52(0.33-0.80)$ & 0.739 \\
\hline$\geqslant 55$ & $0.94(0.73-1.22)$ & $0.76(0.58-0.98)$ & $1.08(0.84-1.38)$ & $0.77(0.60-0.99)$ & 0.294 \\
\hline$P_{\text {strata }}{ }^{d}$ & \multicolumn{5}{|c|}{0.176} \\
\hline \multicolumn{6}{|l|}{ Sex } \\
\hline Female & $0.53(0.32-0.89)$ & $0.33(0.14-0.76)$ & $0.52(0.32-0.86)$ & $0.54(0.27-1.10)$ & 0.677 \\
\hline Male & $1.02(0.82-1.28)$ & $0.80(0.64-1.01)$ & $0.96(0.77-1.20)$ & $0.71(0.50-0.99)$ & 0.015 \\
\hline$P_{\text {strata }} \mathbf{d}$ & \multicolumn{5}{|c|}{0.114} \\
\hline \multicolumn{6}{|l|}{ Education } \\
\hline$\leqslant$ High school graduate & $0.88(0.68-1.13)$ & $0.70(0.54-0.91)$ & $0.95(0.74-1.22)$ & $0.63(0.49-0.81)$ & 0.371 \\
\hline$\geqslant$ Some college & $1.08(0.70-1.65)$ & 0.77 (0.49-1.19) & $0.99(0.65-1.51)$ & $0.96(0.62-1.49)$ & 0.737 \\
\hline$P_{\text {strata }}{ }^{\mathrm{d}}$ & \multicolumn{5}{|c|}{0.486} \\
\hline \multicolumn{6}{|l|}{ Geographic region $^{\text {e }}$} \\
\hline Europe & $0.93(0.68-1.26)$ & $0.83(0.61-1.12)$ & $1.08(0.80-1.44)$ & $0.61(0.45-0.82)$ & 0.201 \\
\hline America & $1.01(0.74-1.38)$ & $0.71(0.52-0.99)$ & $0.97(0.70-1.35)$ & $0.99(0.71-1.39)$ & 0.830 \\
\hline Asia & $1.13(0.57-2.25)$ & $1.07(0.55-2.09)$ & $0.84(0.39-1.82)$ & $0.86(0.41-1.83)$ & NE \\
\hline$P_{\text {strata }}{ }^{d}$ & \multicolumn{5}{|c|}{0.087} \\
\hline \multicolumn{6}{|l|}{ Body mass index } \\
\hline$<25 \mathrm{~kg} \mathrm{~m}^{-2}$ & $1.10(0.79-1.53)$ & $0.87(0.61-1.22)$ & $1.08(0.76-1.53)$ & $0.71(0.50-1.02)$ & 0.695 \\
\hline$\geqslant 25 \mathrm{~kg} \mathrm{~m}^{-2}$ & $0.89(0.66-1.19)$ & $0.70(0.52-0.93)$ & $0.94(0.71-1.24)$ & $0.72(0.54-0.96)$ & 0.118 \\
\hline$P_{\text {strata }}{ }^{d}$ & \multicolumn{5}{|c|}{0.830} \\
\hline \multicolumn{6}{|l|}{ Tobacco consumption } \\
\hline Never user & $1.39(0.63-3.06)$ & $0.89(0.37-2.13)$ & $0.57(0.21-1.52)$ & $0.74(0.30-1.84)$ & 0.793 \\
\hline Former user & $1.00(0.70-1.44)$ & $0.69(0.47-1.01)$ & $1.07(0.75-1.52)$ & $0.73(0.50-1.06)$ & 0.340 \\
\hline Current user & $0.82(0.63-1.08)$ & $0.76(0.58-0.99)$ & $0.74(0.56-0.97)$ & $0.68(0.51-0.90)$ & 0.929 \\
\hline$P_{\text {strata }}{ }^{d}$ & \multicolumn{5}{|c|}{0.616} \\
\hline \multicolumn{6}{|l|}{ Alcohol consumption $^{f}$} \\
\hline Never and light drinker & $0.97(0.68-1.37)$ & $0.65(0.45-0.95)$ & $1.03(0.72-1.49)$ & $0.74(0.50-1.10)$ & 0.223 \\
\hline Moderate drinker & $0.92(0.66-1.29)$ & $0.74(0.53-1.05)$ & $0.86(0.61-1.21)$ & $0.78(0.55-1.11)$ & 0.361 \\
\hline Heavy drinker & $1.00(0.63-1.61)$ & $0.89(0.56-1.43)$ & $1.17(0.76-1.82)$ & $0.62(0.40-0.94)$ & 0.650 \\
\hline$P_{\text {strata }}{ }^{d}$ & \multicolumn{5}{|c|}{0.732} \\
\hline \multicolumn{6}{|c|}{ 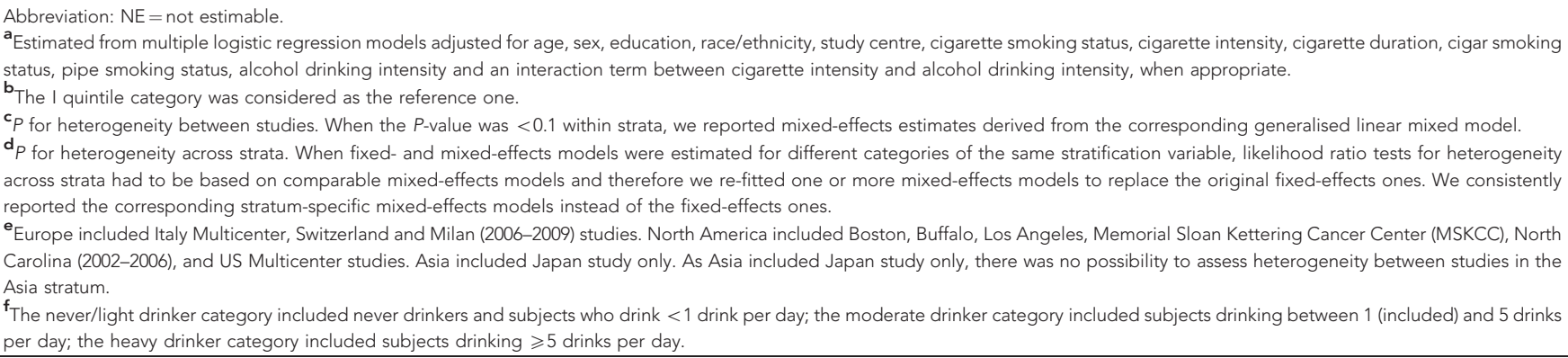 } \\
\hline
\end{tabular}

pharyngeal cancer, and for four of them for laryngeal cancer. In either case, the point estimates were generally in line with the ones from the main analysis, although the ORs tended to be higher (data not shown). Similarly, with the additional adjustment by total fruit or total vegetable intake, the inverse association between vitamin $\mathrm{E}$ and either cancer site persisted, although the ORs were higher than before (for oral and pharyngeal cancer: $\mathrm{OR}=0.70,95 \% \mathrm{CI}$ : 0.58 0.84 for the highest quintile category of vitamin $\mathrm{E}$ and adjustment by total fruit intake, $\mathrm{OR}=0.67,95 \% \mathrm{CI}: 0.56-0.80$ for the adjustment by total vegetable intake; for laryngeal cancer: $\mathrm{OR}=0.80,95 \% \mathrm{CI}: 0.68-0.94$ for the highest quintile category of vitamin $\mathrm{E}$ and adjustment by total fruit intake, $\mathrm{OR}=0.76,95 \% \mathrm{CI}$ : 0.64-0.90 for the adjustment by total vegetable intake) (data not shown). With the additional adjustment by supplemental use of vitamin $\mathrm{E}$ intake, the corresponding ORs for natural vitamin $\mathrm{E}$ intake were in line with the ones obtained without such an adjustment (for oral and pharyngeal cancer: $\mathrm{OR}=0.62$, 95\% CI: 0.50-0.77 for the highest quintile category of vitamin $\mathrm{E}$ intake; for laryngeal cancer: $\mathrm{OR}=0.68,95 \% \mathrm{CI}: 0.56-0.83, P$-values of the likelihood ratio tests of significance of the interaction effect $<0.001$ ) (data not shown).

Supplementary Figure 1 shows the study-specific ORs of oral and pharyngeal combined, and laryngeal cancers and corresponding 95\% CIs for the highest quintile category of vitamin E intake as compared with the lowest one. The ORs of oral and pharyngeal cancers combined were below unity in seven studies (significant in four) and above unity in two studies (nonsignificant); the ORs of laryngeal cancer were below unity in five studies (significant in one) and above unity in three studies (nonsignificant). Results from the influence analysis were reassuring, as the exclusion of one study at a time did not materially change the point estimates. 


\section{DISCUSSION}

The present analysis shows that vitamin $\mathrm{E}$ intake was inversely related to oral and pharyngeal and to laryngeal cancer risk. The identified associations were generally similar across oral and pharyngeal cancer subsites and in strata of major confounding and risk factors. However, some heterogeneity between studies was detected for oral and pharyngeal cancer.

The term 'vitamin E' includes eight different forms of tocopherols and tocotrienols produced by plants: $\alpha-, \beta-, \gamma$ - and $\delta$-tocopherol and $\alpha$-, $\beta$-, $\gamma$ - and $\delta$-tocotrienol. These fat-soluble food components are thought to have a primary role in protecting both low-density lipoproteins and polyunsaturated fatty acids in cell membranes from oxidation, thus acting indirectly on damage to DNA and other cellular molecules. Indeed, some of the photosynthesis-derived reactive oxygen species generated by oxidation of these compounds in membranes, if not deactivated, can diffuse into the nucleus and cause mutagenesis (Seidman et al, 1999). Moreover, compounds with vitamin E activity appear to scavenge oxygen radicals attacking cell membranes and to terminate free radical chain reactions within cell membranes (Schorah, 1995).

Tobacco use per se increases oxidative stress, and therefore enhances the possibility of cancer-causing mutations, oxidisation of lipids and proteins and alteration of signal transduction pathways that damage cells. In heavy alcohol drinkers, the entire nutritional status may be impaired because of malnutrition, and the deficiencies of vitamins and trace elements may contribute to alcohol-associated carcinogenesis. Moreover, the increased oxidative stress observed during ethanol metabolism leads to an increased requirement for glutathion and $\alpha$-tocopherol (Poschl and Seitz, 2004; World Cancer Research Fund/American Institute for Cancer Research, 2007). In the current paper, we found an interaction between tobacco consumption and vitamin $\mathrm{E}$ intake for oral and pharyngeal cancer, with current smokers experiencing a stronger risk reduction at the lowest vitamin $\mathrm{E}$ intakes as compared with former and never smokers. No evidence of effect modification was found for alcohol consumption in either cancer site.

Vitamin $\mathrm{E}$ is part of a synergistic biochemical system involving also vitamin $\mathrm{C}$, the carotenoids, glutathione and selenium that is designed to protect structural and functional lipids, proteins and nucleic acids from oxidation (Shklar and Schwartz, 1993). The synergy between these nutrients as found in whole foods, their derivation from the same food sources and/or the beneficial effects of other phytochemical compounds found in fruit and vegetables may account for the apparent effects of each of these nutrients. In addition, these reasons may explain why cancer risk is more strongly associated with total fruit and vegetable consumption than with any particular nutrient (Pavia et al, 2006; World Cancer Research Fund/ American Institute for Cancer Research, 2007). In our analysis, we investigated the possibility of an interaction effect between vitamin $\mathrm{E}$ intake and nine potentially related nutrients and that with total fruit and total vegetable consumption. No interaction was evident for either oral and pharyngeal cancers combined or laryngeal cancer and, even when suggested by the likelihood ratio tests, the inclusion in the models of an adjustment by an extra nutrient or by total fruit/ vegetable consumption did not materially modify the key finding of an inverse association between higher natural vitamin $\mathrm{E}$ intake and the cancer sites of interest.

Some of the studies included in the present analysis already contributed to separate original reports on vitamin $\mathrm{E}$ intake or provided data for original publications on data partially overlapping with them (McLaughlin et al, 1988; Gridley et al, 1990; Day et al, 1993; Negri et al, 2000; Bidoli et al, 2003; Suzuki et al, 2006; Bravi et al, 2013). Besides them, we are aware of at least seven papers (Riboli et al, 1996; Chainani-Wu, 2002; Lucenteforte et al,
2009) that assess the association between vitamin $\mathrm{E}$ intake from natural sources and HNC and/or its subsites. Among these, one provided results on oral and pharyngeal cancer (Marshall et al, 1992), two on laryngeal cancer (Freudenheim et al, 1992; Esteve et al, 1996), two papers concerned UADTC and their subsites (De Stefani et al, 1999; Wright et al, 2007) and two UADTC overall (Zheng et al, 1995; Kasum et al, 2002), with the evidence spread out over the different HNC subsites. Moreover, the same evidence collected on cancer subsites is generally inconclusive. For oral and pharyngeal cancer, a case-control study from the Western New York area showed a weak but nonsignificant protection for higher vitamin E intakes (Marshall et al, 1992), a case-control study from Uruguay showed an increased but nonsignificant risk (De Stefani et al, 1999) and the Alpha-Tocopherol, Beta-Carotene Cancer Prevention (ATBC) trial showed a nonsignificant inverse association in male smokers (Wright et al, 2007). For laryngeal cancer, the southwestern European study (Esteve et al, 1996) and the ATBC trial (Wright et al, 2007) reported that vitamin $\mathrm{E}$ was related to a significant reduction in risk, whereas the remaining two studies from the United States (Freudenheim et al, 1992) and Uruguay (De Stefani et al, 1999) - found a weak-to-moderate nonsignificant reduction in risk. In addition, in the Iowa Women's Health cohort, an inverse nonsignificant association was found in the original study (Zheng et al, 1995) for total vitamin $\mathrm{E}$ intake (from both diet and supplements) and cancers of the mouth/pharynx/esophagus combined, but no association was observed in its update for dietary vitamin $\mathrm{E}$ intake and UADTC overall (including cancers of the nasopharynx, larynx and stomach) after 14 years of follow-up (Kasum et al, 2002).

Concerning vitamin E supplementation, two US case-control studies in the 1990s provided preliminary evidence for an inverse association between vitamin $\mathrm{E}$ and oral and pharyngeal cancer (Barone et al, 1992; Gridley et al, 1992). However, more recent results from a systematic review and meta-analysis (Bardia et al, 2008) and subsequent original studies (Bairati et al, 2005, 2006; Wright et al, 2007) did not provide support for vitamin E supplementation on the reduced risk of HNC.

Serum levels of $\alpha$-tocopherol were related to oral and/or pharyngeal, and laryngeal cancers in an inconsistent way. The ATBC trial in Finland and two nested case-control studies conducted in Finland and the United States provided opposite inconclusive results for oral and pharyngeal cancer (Knekt et al, 1991; Zheng et al, 1993; Wright et al, 2007). In a case-control study from India, serum levels of $\alpha$-tocopherol were $25 \%$ lower among oral cancer cases than among controls (Krishnamurthy and Jaya, 1986). In Finland, increased serum $\alpha$-tocopherol concentrations were associated with a lower risk of laryngeal cancer in the ATBC study (Wright et al, 2007), but similar findings were nonsignificant in the above-mentioned nested case-control study (Knekt et al, 1991). A nested case-control study on JapaneseAmerican men in Hawaii found no difference in the (adjusted) mean serum levels of $\alpha$-tocopherol among UADTC cases and controls (Nomura et al, 1997).

Our analysis has several strengths. The large sample size allowed to examine the association between vitamin $\mathrm{E}$ intake and $\mathrm{HNC}$ by cancer subsite and within relevant subgroups of the study population with the adequate statistical power. The definition of the outcome variable was accurate and standardised across studies. Similarly, we were able to adequately control for the potential confounding effect of several factors that were harmonised across studies. For tobacco smoking and alcohol drinking - the main risk factors for HNC - we accounted for status, intensity and duration of cigarette smoking, status of cigar and pipe use and intensity of alcohol drinking. We also proposed a sensitivity analysis where we assessed the role of several other nutrients, total fruits, total vegetables and supplemental use of vitamin $\mathrm{E}$ in their interaction with vitamin $\mathrm{E}$. 
However, there are also a few limitations in our work. This analysis suffers from all the issues inherent to a pooled analysis, including, among others, if it is reasonable to pool the studyspecific information on the exposure of interest or not, if some preprocessing/harmonisation of the exposure may help and how to do it, how to clearly identify the potential sources of heterogeneity between studies and which statistical approach is more appropriate for the analysis.

Sources of vitamin E are different across countries. A study based on the same FFQ administered in the Italy Multicenter, Milan (2006-2009), and Switzerland studies showed that vitamin E derived from the following food sources: green salad, spinach/other greens, tomatoes, vegetable soup, 'salad with carrots, cucumbers and peppers', apples/pears, peaches/apricots/prunes, 'pasta/rice with tomato sauce', 'tuna/sardines in oil pack' and 'chicken/turkey, roasted, fried, stewed' ( $\sim 44.5 \%$ cumulative contribution) (Favero et al, 1997). In the Japanese 102-item FFQ from which our brief Japanese FFQ was derived, vitamin E was supplied by different foods and condiments, with well-milled rice, mixed salad oil, chicken eggs, mixed vegetable oil, spinach, mayonnaise, pumpkin, safflower oil, margarine and koji miso (soybean paste) being the top 10 sources $(\sim 53 \%$ cumulative contribution) (Imaeda et al, 1999). Similarly, major contributors of vitamin E in the US diet from the second National Health and Nutrition examination survey (1976-1980) included, among 15 aggregate categories, the following foods and condiments: fats and oils, vegetables, meat/ poultry/fish, desserts, breakfast cereals, fruit, bread/grain products, dairy products, mixed main dishes and nuts/seeds ( $\sim 90 \%$ cumulative contribution), with a major role for highly fortified foods among single items (Murphy et al, 1990).

As compared with food groups, extra imprecision is likely present in the measurement of individual nutrients, because of lack of complete information in the nutrient databases used in their calculation (Willett, 2013). This may result in stronger and more consistent associations for food groups than for the corresponding indexes of nutrient intake.

Stronger associations may be observed for other nutrients than vitamin $\mathrm{E}$ because of the challenge of measuring vitamin $\mathrm{E}$ intake accurately (Byers and Guerrero, 1995). Vitamin E comes largely from foods enriched with vegetable oils that are more difficult to accurately define and measure than foods such as citrus fruit, from which vitamin $\mathrm{C}$ is derived (for instance, cumulative contribution of the top 10 foods contributing mostly to vitamin E: $44.5 \%$ vs $82 \%$ for vitamin C, as to the paper based on the Italy Multicenter-Milan (2006-2009)-Switzerland FFQ (Favero et al, 1997)). Moreover, vitamin $\mathrm{E}$ levels in foods are influenced by processing and preparation methods.

Results of case-control studies are prone to selection and recall bias, and nondifferential misclassification of individual intake might have also occurred because of random measurement error. In addition, although we adjusted our estimates for major recognised risk factors for $\mathrm{HNC}$, uncontrolled confounding from other dietary and nondietary factors cannot be excluded. Concerning smoking and alcohol consumption, we acknowledge that the adjustment variables we used are likely to suffer from mismeasurement to some extent. Oropharyngeal squamous cell carcinoma has also been linked both epidemiologically and molecularly to human papillomavirus (Allen et al, 2010). However, our effect estimates did not materially differ across subsites of oral and pharyngeal cancer.

Because of the many observations, outcomes and subsets that are typically made or addressed in epidemiology, multiple comparisons issue has to be somehow taken into account in reporting results of epidemiological studies (Berry, 2012). In the present study, stratified analyses implied carrying out several tests of significance for oral and pharyngeal cancer and several of them for laryngeal cancer too. If a 0.1 cutoff was considered for a heterogeneity deemed to be significant, adjustment for multiple comparisons is somehow acknowledged, although not formally taken into account through existing solutions.

In conclusion, the present paper indicates a protective role for vitamin $\mathrm{E}$ in cancers of the oral cavity and pharynx as well as larynx. Although sources of vitamin E may be different across countries, this may point to a protective role of foods rich in vitamin $\mathrm{E}$, including vegetable oils, vegetables and eggs, on cancers at the mentioned sites.

\section{ACKNOWLEDGEMENTS}

The INHANCE Pooled Data Project was supported by grants from the National Institutes of Health (NIH), National Cancer Institute, (NCI) R03CA113157 and NIDCR R03DE016611. Individual studies were funded by the following grants: (1) Italy Multicenter study: Italian Association for Research on Cancer (AIRC), Italian League Against Cancer, and Italian Ministry of Research; (2) Swiss study: the Swiss Research against Cancer/Oncosuisse (KFS-700 and OCS-1633); (3) Los Angeles study: NIH (P50CA090388, R01DA011386, R03CA077954, T32CA009142, U01CA096134, R21ES011667) and the Alper Research Program for Environmental Genomics of the UCLA Jonsson Comprehensive Cancer Center; (4) Boston study: NIH (R01CA078609, R01CA100679); (5) US multicenter study: The Intramural Program of the NCI, NIH, USA; (6) MSKCC study: NIH (R01CA051845); (7) Japan study (20012005): Scientific Research grant from the Ministry of Education, Science, Sports, Culture and Technology of Japan (17015052) and grant for the Third-Term Comprehensive 10-Year Strategy for Cancer Control from the Ministry of Health, Labor and Welfare of Japan (H20-002); (8) North Carolina (2002-2006) study; (9) Buffalo study; (10) Milan study (2006-2009): Italian Association for Cancer Research (AIRC Grant Number 10068), Italian Foundation for Cancer Research (FIRC), and Italian Ministry of Education (PRIN 2009 X8YCBN). FT was supported by a fellowship from FIRC.

\section{CONFLICT OF INTEREST}

The authors declare no conflict of interest.

\section{AUTHOR CONTRIBUTIONS}

MH, MF, CLV, PB and AD designed research; KM, DS, CLV, AO, JPZ, DMW, VJ, KM, ZFZ, HM, FL, VE, CB, KK, MM, SS and GPY conducted research and provided single-study databases; SCC and YAL prepared the pooled data set for the analysis; MP provided advice on nutritional issues; VE performed all statistical analyses; VE and FT performed the meta-analysis; VE wrote the paper and had primary responsibility for final content. All authors read and approved the final manuscript.

\section{REFERENCES}

Allen CT, Lewis Jr JS, El-Mofty SK, Haughey BH, Nussenbaum B (2010) Human papillomavirus and oropharynx cancer: biology, detection and clinical implications. Laryngoscope 120: 1756-1762.

Applied Research Program, National Cancer Institute (2005) Diet $^{* C a l c}$ Analysis Program, Version 1.4.3. National Cancer Institute: Bethesda, MD. Bairati I, Meyer F, Gelinas M, Fortin A, Nabid A, Brochet F, Mercier JP, Tetu B, Harel F, Masse B, Vigneault E, Vass S, del Vecchio P, Roy J (2005) A randomized trial of antioxidant vitamins to prevent second primary cancers in head and neck cancer patients. J Natl Cancer Inst 97: 481-488. 
Bairati I, Meyer F, Jobin E, Gelinas M, Fortin A, Nabid A, Brochet F, Tetu B (2006) Antioxidant vitamins supplementation and mortality: a randomized trial in head and neck cancer patients. Int J Cancer 119: 2221-2224.

Bardia A, Tleyjeh IM, Cerhan JR, Sood AK, Limburg PJ, Erwin PJ, Montori VM (2008) Efficacy of antioxidant supplementation in reducing primary cancer incidence and mortality: systematic review and metaanalysis. Mayo Clin Proc 83: 23-34.

Barone J, Taioli E, Hebert JR, Wynder EL (1992) Vitamin supplement use and risk for oral and esophageal cancer. Nutr Cancer 18: 31-41.

Bates D, Maechler M, Bolker B (2011) lme4: linear mixed-effects models using S4 classes. URL http://CRAN.R-project.org/package $=$ lme4R package version 0.999375-42.

Baumeister P, Korn G, Berghaus A, Matthias C, Harreus U (2009) Chemopreventive action of dexamethasone and alpha-tocopherol in oxidative stressed cells. Cancer Detect Prev 32: 452-457.

Berry D (2012) Multiplicities in cancer research: ubiquitous and necessary evils. J Natl Cancer Inst 104: 1124-1132.

Bidoli E, Bosetti C, La Vecchia C, Levi F, Parpinel M, Talamini R, Negri E, Maso LD, Franceschi S (2003) Micronutrients and laryngeal cancer risk in Italy and Switzerland: a case-control study. Cancer Causes Control 14: 477-484.

Bjelakovic G, Nikolova D, Gluud LL, Simonetti RG, Gluud C (2012) Antioxidant supplements for prevention of mortality in healthy participants and patients with various diseases. Cochrane Database Syst Rev 3: CD007176.

Block G (1992) The data support a role for antioxidants in reducing cancer risk. Nutr Rev 50: 207-213.

Blot WJ, McLaughlin JK, Winn DM, Austin DF, Greenberg RS, Preston-Martin S, Bernstein L, Schoenberg JB, Stemhagen A, Fraumeni Jr JF (1988) Smoking and drinking in relation to oral and pharyngeal cancer. Cancer Res 48: 3282-3287.

Bosetti C, Gallus S, Trichopoulou A, Talamini R, Franceschi S, Negri E, La Vecchia C (2003) Influence of the Mediterranean diet on the risk of cancers of the upper aerodigestive tract. Cancer Epidemiol Biomarkers Prev 12: 1091-1094.

Bravi F, Bosetti C, Filomeno M, Levi F, Garavello W, Galimberti S, Negri E, La Vecchia C (2013) Foods, nutrients and the risk of oral and pharyngeal cancer. Br J Cancer 109: 2904-2910.

Bravi F, Edefonti V, Randi G, Ferraroni M, La Vecchia C, Decarli A (2012) Dietary patterns and upper aerodigestive tract cancers: an overview and review. Ann Oncol 23: 3024-3029.

Byers T, Guerrero N (1995) Epidemiologic evidence for vitamin C and vitamin E in cancer prevention. Am J Clin Nutr 62: 1385S-1392S.

Chainani-Wu N (2002) Diet and oral, pharyngeal, and esophageal cancer. Nutr Cancer 44: 104-106.

Chen LH, Boissonneault GA, Glauert HP (1988) Vitamin C, vitamin E and cancer (review). Anticancer Res 8: 739-748.

Chuang SC, Jenab M, Heck JE, Bosetti C, Talamini R, Matsuo K, Castellsague X, Franceschi S, Herrero R, Winn DM, La Vecchia C, Morgenstern H, Zhang ZF, Levi F, Dal Maso L, Kelsey K, McClean MD, Vaughan T, Lazarus P, Muscat J, Ramroth H, Chen C, Schwartz SM, Eluf-Neto J, Hayes RB, Purdue M, Boccia S, Cadoni G, Zaridze D, Koifman S, Curado MP, Ahrens W, Benhamou S, Matos E, Lagiou P, Szeszenia-Dabrowska N, Olshan AF, Fernandez L, Menezes A, Agudo A, Daudt AW, Merletti F, Macfarlane GJ, Kjaerheim K, Mates D, Holcatova I Schantz S, Yu GP, Simonato L, Brenner H, Mueller H, Conway DI, Thomson P, Fabianova E, Znaor A, Rudnai P, Healy CM, Ferro G, Brennan P, Boffetta P, Hashibe M (2012) Diet and the risk of head and neck cancer: a pooled analysis in the INHANCE consortium. Cancer Causes Control 23: 69-88.

Conway DI, Hashibe M, Boffetta P, Wunsch-Filho V, Muscat J, La Vecchia C, Winn DM (2009) Enhancing epidemiologic research on head and neck cancer: INHANCE - The international head and neck cancer epidemiology consortium. Oral Oncol 45: 743-746.

Cui Y, Morgenstern H, Greenland S, Tashkin DP, Mao J, Cao W, Cozen W, Mack TM, Zhang ZF (2006) Polymorphism of Xeroderma Pigmentosum group $\mathrm{G}$ and the risk of lung cancer and squamous cell carcinomas of the oropharynx, larynx and esophagus. Int J Cancer 118: 714-720.

Day GL, Blot WJ, Austin DF, Bernstein L, Greenberg RS, Preston-Martin S, Schoenberg JB, Winn DM, McLaughlin JK, Fraumeni Jr. JF (1993) Racial differences in risk of oral and pharyngeal cancer: alcohol, tobacco, and other determinants. J Natl Cancer Inst 85: 465-473.
Deeks JJ, Higgins JPT, Altman DG (eds) (2011) Chapter 9: Analysing data and undertaking meta-analyses. In: Higgins JPT, Green S (eds) Cochrane Handbook for Systematic Reviews of Interventions Version 5.1.0 (updated March 2011). The Cochrane Collaboration. Available from www.cochrane-handbook.org (last accessed on 15 March 2015).

De Stefani E, Ronco A, Mendilaharsu M, Deneo-Pellegrini H (1999) Diet and risk of cancer of the upper aerodigestive tract-II. Nutrients. Oral Oncol 35: 22-26.

DietSys (1999) HHHQ-DietSys Analysis Software, Version 4.02. National Cancer Institute: Bethesda, MD. Available from http:// appliedresearch.cancer.gov/DietSys/materials.html.

Divaris K, Olshan AF, Smith J, Bell ME, Weissler MC, Funkhouser WK, Bradshaw PT (2010) Oral health and risk for head and neck squamous cell carcinoma: the Carolina Head and Neck Cancer Study. Cancer Causes Control 21: 567-575.

Dresser CM (1983) From nutrient data to a data base for a health and nutrition examination survey. Organization, coding and values-real or imputed. Proceeding of the 8th National Nutrient Data Base Conference, Minneapolis, MN, USA, 92-104.

Edefonti V, Bravi F, Garavello W, La Vecchia C, Parpinel M, Franceschi S, Dal Maso L, Bosetti C, Boffetta P, Ferraroni M, Decarli A (2010a) Nutrient-based dietary patterns and laryngeal cancer: evidence from an exploratory factor analysis. Cancer Epidemiol Biomarkers Prev 19: 18-27.

Edefonti V, Bravi F, La Vecchia C, Randi G, Ferraroni M, Garavello W, Franceschi S, Talamini R, Boffetta P, Decarli A (2010b) Nutrient-based dietary patterns and the risk of oral and pharyngeal cancer. Oral Oncol 46: 343-348.

Edefonti V, Hashibe M, Ambrogi F, Parpinel M, Bravi F, Talamini R, Levi F, Yu G, Morgenstern H, Kelsey K, McClean M, Schantz S, Zhang Z, Chuang S, Boffetta P, La Vecchia C, Decarli A (2012) Nutrient-based dietary patterns and the risk of head and neck cancer: a pooled analysis in the International Head and Neck Cancer Epidemiology consortium. Ann Oncol 23: 1869-1870.

Edefonti V, Hashibe M, Parpinel M, Turati F, Serraino D, Matsuo K, Olshan AF, Zevallos JP, Winn DM, Moysich K, Zhang ZF, Morgenstern H, Levi F, Kelsey K, McClean M, Bosetti C, Galeone C, Schantz S, Yu GP, Boffetta P, Lee YCA, Chuang SC, La Vecchia C, Decarli A (2014) Natural vitamin $\mathrm{C}$ intake and the risk of head and neck cancer: a pooled analysis in the International Head and Neck Cancer Epidemiology consortium. Int J Cancer. E-pub ahead of print.

Esteve J, Riboli E, Pequignot G, Terracini B, Merletti F, Crosignani P, Ascunce N, Zubiri L, Blanchet F, Raymond L, Repetto F, Tuyns AJ (1996) Diet and cancers of the larynx and hypopharynx: the IARC multi-center study in southwestern Europe. Cancer Causes Control 7: 240-242.

Favero A, Salvini S, Russo A, Parpinel M, Negri E, Decarli A, La Vecchia C, Giacosa A, Franceschi S (1997) Sources of macro- and micronutrients in Italian women: results from a food frequency questionnaire for cancer studies. Eur J Cancer Prev 6: 277-277.

Flagg EW, Coates RJ, Greenberg RS (1995) Epidemiologic studies of antioxidants and cancer in humans. J Am Coll Nutr 14: 419-427.

Freudenheim JL, Graham S, Byers TE, Marshall JR, Haughey BP, Swanson MK, Wilkinson G (1992) Diet, smoking, and alcohol in cancer of the larynx: a case-control study. Nutr Cancer 17: 33-45.

Galeone C, Edefonti V, Parpinel M, Leoncini E, Matsuo K, Talamini R, Olshan AF, Zevallos JP, Winn DM, Jayaprakash V, Moysich K, Zhang ZF, Morgenstern H, Levi F, Bosetti C, Kelsey K, McClean M, Schantz S, Yu GP, Boffetta P, Lee YC, Hashibe M, La Vecchia C, Boccia S (2014) Folate intake and the risk of oral cavity and pharyngeal cancer: A pooled analysis within the International Head and Neck Cancer Epidemiology Consortium. Int J Cancer 136(4): 904-914.

Garewal H (1995) Antioxidants in oral cancer prevention. Am J Clin Nutr 62 : 1410S-1416S.

Gnagnarella P, Salvini S, Parpinel M (2008) Food composition database for epidemiological studies in Italy. Version 2. Available from http:// www.bda-ieo.it.

Gridley G, McLaughlin JK, Block G, Blot WJ, Gluch M, Fraumeni Jr JF (1992) Vitamin supplement use and reduced risk of oral and pharyngeal cancer. Am J Epidemiol 135: 1083-1092.

Gridley G, McLaughlin JK, Block G, Blot WJ, Winn DM, Greenberg RS, Schoenberg JB, Preston-Martin S, Austin DF, Fraumeni Jr JF (1990) Diet and oral and pharyngeal cancer among blacks. Nutr Cancer 14: 219-225.

Hashibe M, Brennan P, Benhamou S, Castellsague X, Chen C, Curado MP, Dal Maso L, Daudt AW, Fabianova E, Fernandez L, Wunsch-Filho V, 
Franceschi S, Hayes RB, Herrero R, Koifman S, La Vecchia C, Lazarus P, Levi F, Mates D, Matos E, Menezes A, Muscat J, Eluf-Neto J, Olshan AF, Rudnai P, Schwartz SM, Smith E, Sturgis EM, Szeszenia-Dabrowska N, Talamini R, Wei Q, Winn DM, Zaridze D, Zatonski W, Zhang ZF, Berthiller J, Boffetta P (2007) Alcohol drinking in never users of tobacco, cigarette smoking in never drinkers, and the risk of head and neck cancer: pooled analysis in the International Head and Neck Cancer Epidemiology Consortium. J Natl Cancer Inst 99: 777-779.

Hosmer DW, Lemeshow S (2000) Applied Logistic Regression, 2nd edn. John Wiley \& Sons, Inc.: New York, NY.

Imaeda N, Tokudome Y, Ikeda M, Kitagawa I, Fujiwara N, Tokudome S (1999) Foods contributing to absolute intake and variance in intake of selected vitamins, minerals and dietary fiber in middle-aged Japanese. J Nutr Sci Vitaminol (Tokyo) 45: 519-522.

Jayaprakash V, Rigual NR, Moysich KB, Loree TR, Nasca MA, Menezes RJ, Reid ME (2006) Chemoprevention of head and neck cancer with aspirin a case-control study. Arch Otolaryngol Head Neck Surg 132: 1231-1236.

Kasum CM, Jacobs Jr. DR, Nicodemus K, Folsom AR (2002) Dietary risk factors for upper aerodigestive tract cancers. Int J Cancer 99: 267-272.

Knekt P, Aromaa A, Maatela J, Alfthan G, Aaran RK, Nikkari T, Hakama M, Hakulinen T, Teppo L (1991) Serum micronutrients and risk of cancers of low incidence in Finland. Am J Epidemiol 134: 356-361.

Krishnamurthy S, Jaya S (1986) Serum alpha-tocopherol, lipo-peroxides, and ceruloplasmin and red cell glutathione and antioxidant enzymes in patients of oral cancer. Indian J Cancer 23: 36-42.

La Vecchia C, Decarli A, Serafini M, Parpinel M, Bellocco R, Galeone C, Bosetti C, Zucchetto A, Polesel J, Lagiou P, Negri E, Rossi M (2013) Dietary total antioxidant capacity and colorectal cancer: a large casecontrol study in Italy. Int J Cancer 133: 1447-1451.

Leoncini E, Edefonti V, Hashibe M, Parpinel M, Ferraroni M, Serraino D, Matsuo K, Olshan AF, Zevallos JP, Winn DM, Moysich K, Zhang ZF, Morgenstern H, Levi F, Kelsey K, McClean M, Bosetti C, Schantz S, Yu GP, Boffetta P, Lee YCA, Chuang SC, Decarli A, La Vecchia C, Boccia S (2015) Carotenoid intake and head and neck cancer: a pooled analysis in the International Head and Neck Cancer Epidemiology Consortium. Eur J Epidemiol. in press.

Levi F, Pasche C, La Vecchia C, Lucchini F, Franceschi S, Monnier P (1998) Food groups and risk of oral and pharyngeal cancer. Int J Cancer 77: 705-709.

Li Q, Chuang SC, Eluf-Neto J, Menezes A, Matos E, Koifman S, Wunsch-Filho V, Fernandez L, Daudt AW, Curado MP, Winn DM, Franceschi S, Herrero R, Castellsague X, Morgenstern H, Zhang ZF, Lazarus P, Muscat J, McClean M, Kelsey KT, Hayes RB, Purdue MP, Schwartz SM, Chen C, Benhamou S, Olshan AF, Yu G, Schantz S, Ferro G, Brennan P, Boffetta P, Hashibe M (2012) Vitamin or mineral supplement intake and the risk of head and neck cancer: pooled analysis in the INHANCE consortium. Int J Cancer 131: 1686-1689.

Lucenteforte E, Garavello W, Bosetti C, La Vecchia C (2009) Dietary factors and oral and pharyngeal cancer risk. Oral Oncol 45: 461-467.

Marshall JR, Graham S, Haughey BP, Shedd D, O'Shea R, Brasure J, Wilkinson GS, West D (1992) Smoking, alcohol, dentition and diet in the epidemiology of oral cancer Eur J Cancer B. Oral Oncol 28B: 9-15.

McLaughlin JK, Gridley G, Block G, Winn DM, Preston-Martin S, Schoenberg JB, Greenberg RS, Stemhagen A, Austin DF, Ershow AG et al. (1988) Dietary factors in oral and pharyngeal cancer. J Natl Cancer Inst 80: 1237-1243.

Murphy SP, Subar AF, Block G (1990) Vitamin E intakes and sources in the United States. Am J Clin Nutr 52: 361-367.

Negri E, Franceschi S, Bosetti C, Levi F, Conti E, Parpinel M, La Vecchia C (2000) Selected micronutrients and oral and pharyngeal cancer. Int J Cancer 86: 122-127.

Nomura AM, Ziegler RG, Stemmermann GN, Chyou PH, Craft NE (1997) Serum micronutrients and upper aerodigestive tract cancer. Cancer Epidemiol Biomarkers Prev 6: 407-412.

Pavia M, Pileggi C, Nobile CG, Angelillo IF (2006) Association between fruit and vegetable consumption and oral cancer: a meta-analysis of observational studies. Am J Clin Nutr 83: 1126-1134.

Peters ES, McClean MD, Liu M, Eisen EA, Mueller N, Kelsey KT (2005) The ADH1C polymorphism modifies the risk of squamous cell carcinoma of the head and neck associated with alcohol and tobacco use. Cancer Epidemiol Biomarkers Prev 14: 476-482.
Pinheiro JC, Bates DM (2000) Mixed-Effects Models in S and S-PLUS. Springer-Verlag: New York, NY.

Poschl G, Seitz HK (2004) Alcohol and cancer. Alcohol Alcohol 39: 155-155.

R Core Team (2014) R: A Language and Environment for Statistical Computing. R Foundation for Statistical Computing: Vienna, Austria. Available from http://www.R-project.org.

Resource Council, Science and Technology Agency, the Government of Japan (2000) Standard Tables of Food Composition in Japan, 5th Revised Version (in Japanese with English translation). Ministry of Finance Printing Bureau: Tokyo, Japan.

Riboli E, Kaaks R, Esteve J (1996) Nutrition and laryngeal cancer. Cancer Causes Control 7: 147-156.

Salvini S, Parpinel M, Gnagnarella P, Maisonneuve P, Turrini A (1998) Banca Dati di Composizione Degli Alimenti per Studi Epidemiologici in Italia. Istituto Europeo di Oncologia: Milano, Italy.

Schantz SP, Zhang ZF, Spitz MS, Sun M, Hsu TC (1997) Genetic susceptibility to head and neck cancer: interaction between nutrition and mutagen sensitivity. Laryngoscope 107: 765-771.

Schorah CJ (1995) Micronutrients, antioxidants and risk of cancer. Bibl Nutr Dieta 52: 92-107.

Scott DW (2005) Multivariate Density Estimation. Theory, Practice and Visualization. Wiley: New York, NY.

Seidman MD, Quirk WS, Shirwany NA (1999) Reactive oxygen metabolites, antioxidants and head and neck cancer. Head Neck 21: 467-469.

Shklar G, Schwartz J (1993) Oral cancer inhibition by micronutrients. The experimental basis for clinical trials. Eur J Cancer B Oral Oncol 29B: 9-16.

Suzuki T, Wakai K, Matsuo K, Hirose K, Ito H, Kuriki K, Sato S, Ueda R, Hasegawa Y, Tajima K (2006) Effect of dietary antioxidants and risk of oral, pharyngeal and laryngeal squamous cell carcinoma according to smoking and drinking habits. Cancer Sci 97: 760-767.

U.S. Department of Agriculture, Agricultural Research Service (1993) Composition of foods raw, processed, prepared. Agriculture Handbook 8 , 1-21 and supplements.

U.S. Department of Agriculture, Agricultural Research Service (2013) USDA National nutrient database for standard reference, Release 26 and previous versions. Nutrient Data Laboratory Home Page. Available from http:// www.ars.usda.gov/ba/bhnrc/ndl.

U.S. Department of Health and Human Services, National Center for Health Statistics (1982) Public Use Data Tape Documentation: Model Gram and Nutrient Composition (Computer Tape 5702 and 5703). U.S. Government Printing Office: Hyattsville, MD.

Venables WN, Ripley BD (2002) Modern Applied Statistics with S, 4th edn. Springer New York, NY. Available from http://www.stats.ox.ac.uk/pub/ MASS4.

Willett W (2013) Nutritional Epidemiology, 3th edn. Oxford University Press: New York.

Willett W, Stampfer MJ (1986) Total energy intake: implications for epidemiologic analyses. Am J Epidemiol 124: 17-27.

World Cancer Research Fund/American Institute for Cancer Research (2007) Food, Nutrition, Physical Activity and the Prevention of Cancer: A Global Perspective. AICR: Washington, DC.

Wright ME, Virtamo J, Hartman AM, Pietinen P, Edwards BK, Taylor PR, Huttunen JK, Albanes D (2007) Effects of alpha-tocopherol and betacarotene supplementation on upper aerodigestive tract cancers in a large, randomized controlled trial. Cancer 109: 891-898.

Zheng T, Boyle P, Willett WC, Hu H, Dan J, Evstifeeva TV, Niu S, MacMahon B (1993) A case-control study of oral cancer in Beijing, People's Republic of China. Associations with nutrient intakes, foods and food groups. Eur J Cancer B Oral Oncol 29B: 45-55.

Zheng W, Sellers TA, Doyle TJ, Kushi LH, Potter JD, Folsom AR (1995) Retinol, antioxidant vitamins, and cancers of the upper digestive tract in a prospective cohort study of postmenopausal women. Am J Epidemiol 142: 955-960.

This work is published under the standard license to publish agreement. After 12 months the work will become freely available and the license terms will switch to a Creative Commons AttributionNonCommercial-Share Alike 4.0 Unported License.

Supplementary Information accompanies this paper on British Journal of Cancer website (http://www.nature.com/bjc) 